\title{
Síndrome de Burnout em gestores municipais da saúde
}

\section{Burnout Syndrome in municipal health managers}

\author{
Nelo Augusto Poletto ${ }^{1}$, Livia Fernandes Probst ${ }^{1}$, Tátila Lima de Oliveira ${ }^{1}$, \\ Luciane Miranda Guerra1, Gláucia Maria Bovi Ambrosano1, \\ Karine Laura Cortellazzi ${ }^{1}$, Pedro Rafael Gil-Monte², \\ Rosana de Fátima Possobon ${ }^{1}$
}

\begin{abstract}
Resumo
Introdução: Os gestores municipais da saúde devem responder às demandas de suas equipes e dos pacientes do Sistema Único de Saúde, enfrentando cotidianamente situações potencialmente estressoras. A sobrecarga no trabalho pode levar à exaustão física e mental, com esgotamento dos recursos emocionais, e desencadear a Síndrome de Burnout (SB), cujas consequências podem atingir o serviço gerenciado, uma vez que, ao desempenhar essa atividade laboral, tomam-se decisões determinantes nas ações em saúde que afetam muitas pessoas. Este estudo verificou a presença da $S B$ nos gestores municipais de saúde do Estado de São Paulo. Métodos: Estudo do tipo transversal no qual foram coletados dados socioeconômicos e demográficos. Os níveis de SB foram avaliados pela aplicação do Cuestionario para la Evaluación del Síndrome de Quemarse por el Trabajo (CESQT). A análise descritiva foi realizada por meio do cálculo de frequências. Resultados: Verificou-se baixa prevalência de $S B$ entre os 199 gestores participantes do estudo. Porém houve casos indicativos da presença da SB, inclusive com características de Perfil 2. Conclusão: Esses achados evidenciam que essa classe de profissionais necessita de atenção e cuidados para prevenção e controle das consequências da SB, a fim de evitar que pessoas doentes gerenciem serviços de saúde.

Palavras-chave: esgotamento profissional; gestor de saúde; Sistema Único de Saúde; saúde do trabalhador.
\end{abstract}

\begin{abstract}
Background: Municipal health managers must respond to the demands of their staff and Unified Health System's patients, facing daily potentially stressful situations. The work overload can lead to physical and mental exhaustion, with depletion of emotional resources, and trigger the Burnout Syndrome (BS) with consequences that can affect the service, as the managing activity takes major decisions on health actions that affect many people. This study verified the presence of BS in the municipal health managers in the state of São Paulo. Methods: Cross-sectional study. Demographic and socioeconomic data were collected and BS levels were evaluated by application of the Cuestionario para La Evalución Del Síndrome de Quermarse por El Trabajo (CESQT). The descriptive analysis was performed using the frequency estimation. Results: We verified a low prevalence of BS among 199 participants. However, some cases, including profile 2 features, indicated the presence of BS. Conclusion: These findings show that this is a professional class in need of attention and care to prevent and control the consequences of BS, in order to prevent sickness in health services managers.
\end{abstract}

Keywords: burnout, professional; health manager; Unified Health System; occupational health.

\footnotetext{
${ }^{1}$ Departamento de Odontologia Social, Faculdade de Odontologia de Piracicaba (FOP), Universidade Estadual de Campinas (UNICAMP) - Piracicaba (SP), Brasil. ${ }^{2}$ Universitat de València (UV) - València, Espanha.

Trabalho realizado na Faculdade de Odontologia de Piracicaba (FOP), Universidade Estadual de Campinas (UNICAMP) - Piracicaba (SP), País.

Endereço para correspondência: Rosana de Fátima Possobon - CP 52 - CEP: 13414-903 - Piracicaba (SP), Brasil - Email: possobon@fop.unicamp.br

Fonte de financiamento: nenhuma.

Conflito de interesses: nada a declarar.
} 


\section{INTRODUÇÃO}

A competência em obter sucesso no gerenciamento de mudanças parece ser o grande desafio do líder contemporâneo. Ao gestor cabe a responsabilidade de encontrar alternativas para administrar tais mudanças, adaptando-se às novas realidades organizacionais. Percebe-se, entretanto, que nem sempre os gestores estão preparados para responder às demandas de suas equipes, o que pode resultar em conflitos desgastantes para os empregados, para os próprios gestores e, como não poderia deixar de ser, para a organização ${ }^{1}$.

Os compromissos inerentes ao gestor público em saúde são múltiplos e incluem: planejar, organizar, controlar, avaliar e executar as ações e os serviços de saúde pública e privada; gerenciar a política de recursos humanos e de financiamento em âmbito local e regional; garantir o acesso aos usuários, a equidade e a integralidade das ações e serviços de saúde; garantir a participação popular com o controle social por meio do conselho municipal da saúde e conselhos gestores regionalizados ${ }^{2}$. O gestor em saúde atua na linha de combate do setor, tendo, de um lado, o usuário e suas demandas de saúde e, do outro, seus superiores, com suas limitações orçamentárias e estratégias de gestão que podem não colimar com as perspectivas do gestor. Diante dessas questões, evidencia-se que a natureza de seu trabalho, bem como o contexto em que suas funções laborais são exercidas, o expõe a uma série de potenciais agentes estressores.

A sobrecarga no trabalho pode acarretar exaustão física e mental, baixa realização profissional, levando ao esgotamento dos recursos emocionais do indivíduo. Essa sobrecarga, quando persistente, pode desencadear a chamada Sindrome de Burnout $(S B)$ ou outros problemas psicossomáticos ${ }^{3}$. A $S B$ ocorre em indivíduos cujas profissões os expõem à tensão e ao estresse intensos ${ }^{4}$, e pode ser definida como uma resposta ao estresse laboral crônico, característica dos profissionais que trabalham com pessoas ${ }^{5,6}$. Os portadores de $S B$ apresentam deterioração cognitiva (perda de motivação e baixa realização pessoal no trabalho - baixa ilusão pelo trabalho) e afetiva (esgotamento emocional e físico - desgaste psíquico) e, consequentemente, passam a desenvolver atitudes e condutas negativas com os clientes e organização (comportamentos de indiferença, frieza e distanciamento - indolência). O sentimento de culpa surge subsequentemente a esses sintomas, mas não ocorre necessariamente em todos os indivíduos ${ }^{6}$.

Distinguem-se, portanto, dois perfis no processo de $S B$. O Perfil 1 refere-se ao surgimento de um conjunto de sentimentos e condutas vinculados ao estresse laboral, que dá origem a uma forma moderada de mal-estar, mas que não incapacita o indivíduo para o exercício do seu trabalho, ainda que ele pudesse realizá-lo de forma melhor. Esse perfil caracteriza-se pela presença de baixos níveis de ilusão pelo trabalho e altos níveis de desgaste psíquico e indolência. O Perfil 2 define os casos clínicos mais deteriorados pelo desenvolvimento da $S B$, incluindo, além dos sintomas já mencionados, sentimentos de culpa ${ }^{6}$.

Na literatura atual, não são encontrados estudos que avaliem a $S B$ em gestores de saúde. Assim, este estudo inédito pretende verificar a presença da $S B$ nos gestores municipais em saúde do Estado de São Paulo, reconhecendo as consequências que essa síndrome pode trazer a esses profissionais, uma vez que, no desempenho dessa atividade laboral, o profissional toma decisões determinantes nas ações em saúde que poderão afetar a vida de muitas pessoas.

Considera-se que, na medida em que se entende melhor a $S B$ como processo, identificando suas etapas e dimensões, seus estressores mais importantes, especificamente a cada classe profissional e seus modelos explicativos, podem ser formuladas ações que permitam prevenir, diminuir ou até interrompê-la. Dessa forma, é possível auxiliar o gestor em saúde a prosseguir concretizando seu projeto de vida pessoal e profissional, com o mínimo de estresse.

\section{MÉTODOS}

Trata-se de estudo quantitativo, de prevalência, com base em questionários, envolvendo gestores municipais em saúde do Estado de São Paulo, atuantes no segundo semestre do ano de 2013. O projeto desta pesquisa foi aprovado pelo Comitê de Ética em Pesquisa da Faculdade de Odontologia de Piracicaba-Universidade Estadual de Campinas (FOP-UNICAMP) (Protocolo no 059/2013).

\section{Caracterização do local do estudo e amostra}

O Estado de São Paulo tem 645 municípios, e em todos eles há um gestor em saúde. Desses municípios, 403 têm até 20 mil habitantes, 167 têm de 20 mil a 100 mil habitantes, 36 têm de 100 mil a 200 mil habitantes e 39 têm acima de 200 mil habitantes.

Todos os 645 gestores municipais de saúde do Estado de São Paulo em atividade à época do desenvolvimento da pesquisa foram contatados via mensagem eletrônica, por meio dos endereços eletrônicos (e-mail) fornecidos pelo Conselho de Secretários Municipais de Saúde do Estado de São Paulo "Dr. Sebastião de Moraes" (COSEMS) e, assim, convidados a participar da pesquisa. Além do convite eletrônico, os apoiadores do COSEMS, por solicitação do pesquisador, também estimulavam os gestores a participar do estudo durante as reuniões periódicas (mensais) entre os membros de cada região de saúde (Comissão Intergestores Regional) do Estado de São Paulo.

Ao aceitar o convite, o gestor acessava um link que remetia ao Termo de Consentimento Livre e Esclarecido (TCLE) e deveria concordar com os termos desse documento antes de iniciar o preenchimento dos questionários de coleta de dados. 


\section{Coleta de dados e instrumentos utilizados}

Após a assinatura do TCLE, o gestor tinha acesso ao material de coleta de dados, composto por três instrumentos: um para investigação dos dados demográficos, outro para investigação dos dados socioeconômicos e o terceiro para verificação da presença de esgotamento profissional. O preenchimento dos formulários foi feito de forma on-line, e o prazo máximo para o preenchimento dos questionários foi de 120 dias.

$\mathrm{O}$ instrumento utilizado para coletar informações a respeito das características demográficas era composto por questões estruturadas para identificar gênero, idade, estado civil, número de filhos, grau de instrução, profissão, exercício de outra atividade profissional, além da gestão, tempo de trabalho como gestor e número de horas trabalhadas por dia.

O segundo instrumento, baseado em Meneghim et al. ${ }^{7}$, investigou informações a respeito das características socioeconômicas do gestor, tais como renda familiar mensal, número de pessoas residentes na mesma casa e tipo de moradia.

O terceiro instrumento utilizado foi o "Cuestionario para la Evaluación del Síndrome de Quemarse por el Trabajo" (CESQT), elaborado por Gil-Monte ${ }^{6}$ e validado para a população brasileira por Gil-Monte et al. ${ }^{8}$, adaptado para este estudo, uma vez que, originalmente, foi desenvolvido para aplicação em docentes.

O CESQT é formado por 20 itens, distribuídos em quatro dimensões:

(1) Ilusão pelo trabalho, definida como a expectativa do indivíduo em alcançar determinadas metas laborais, pois isso supõe uma fonte de realização pessoal e profissional (cinco itens);

(2) Desgaste psíquico, definido como a presença de esgotamento emocional e físico decorrente da atividade de trabalho, tendo em vista a necessidade de se relacionar diariamente com pessoas que possuem ou gerem problemas (quatro itens);

(3) Indolência, definida como a presença de atitudes negativas de indiferença e cinismo com os clientes da organização (seis itens);

(4) Culpa, definida como a ocorrência de sentimentos de culpa pelo comportamento e atitudes negativas desenvolvidas no trabalho, principalmente com as pessoas com as quais o trabalhador deve relacionar-se profissionalmente (cinco itens).

A frequência das respostas, neste instrumento, é avaliada por meio de uma escala de frequência de 5 pontos, variando de 0 (nunca) até 4 (muito frequentemente, todos os dias). Cada subescala foi calculada pela média da pontuação dos itens que a compunham. Para classificar os pacientes no Perfil 2, muito baixa pontuação em ilusão e pontuações críticas em desgaste e em indolência devem estar acompanhados de pontuações críticas de culpa ${ }^{6,9}$.

\section{Análise de dados}

Os dados socioeconômicos e demográficos foram descritos em porcentagem para caracterização da amostra.

Para analisar o nível da $S B$ apresentado pela amostra, foram utilizados os cinco níveis propostos por Gil-Monte ${ }^{9}$, de acordo com os percentis 10, 33, 66 e 90 (P10, P33, P66 e P90). Dessa forma, foram classificados como: muito baixo - pontuações menores ou iguais ao P10; baixo - pontuações menores ou iguais ao P33; médio - pontuações menores ou iguais ao P66; alto - pontuações menores que P90; e crítico - pontuações maiores ou iguais ao P90.

Para identificar os casos de Perfil 1 e Perfil 2, foram utilizados os critérios do manual do CESQT ${ }^{9}$. São considerados Perfil 1 os casos que apresentaram pontuações iguais ou superiores a P90 na pontuação média dos 15 itens que formam as subescalas de ilusão pelo trabalho (invertida), desgaste psíquico e indolência, mas inferiores a P90 na subescala de culpa. No Perfil 2 foram incluídos os casos com pontuações iguais ou superiores a P90 na pontuação média dos 15 itens citados e também iguais ou superiores a P90 na subescala de culpa.

\section{RESULTADOS}

Foram excluídos da amostra os gestores que responderam incorretamente ou de forma incompleta os questionários. Dessa forma, a taxa de resposta final foi de $30,9 \%$, ou seja, dos 645 gestores convidados, 199 retornaram os questionários corretamente preenchidos e dentro do prazo estipulado.

A Tabela 1 mostra as informações a respeito dos dados socioeconômicos e demográficos dos participantes. A maioria dos participantes era do gênero feminino (61,3\%). Mais da metade da amostra (52,3\%) estava na faixa etária entre 35 e 50 anos (média de idade de 43 anos). A maioria era casada $(65,3 \%)$ e $74,8 \%$ tinham filhos. A renda de $60,8 \%$ dos participantes era superior a sete salários mínimos vigentes na época da pesquisa. Além disso, $81,4 \%$ possuíam residência própria, $92,5 \%$ moravam com até quatro pessoas e $86,4 \%$ tinham concluído o curso de graduação superior.

As informações a respeito das profissões dos participantes estão apresentadas na Tabela 2 e mostram que 66,3\% eram de áreas da saúde, dos quais a maioria era formada por enfermeiros, cirurgiões-dentistas e médicos.

Em relação à sua atuação profissional como gestor, 59,8\% (mais da metade da amostra) estavam em municípios com até 20 mil habitantes, 53,3\% exerciam a função de gestor havia menos de um ano e 70,3\% trabalhavam mais de 8 h diárias (Tabela 3).

\section{Síndrome de Burnout}

A Tabela 4 apresenta as médias, o desvio-padrão (DP), a variação e a consistência interna ( $\alpha$ de Cronbach) das dimensões do questionário utilizado neste estudo. Todas as dimensões do questionário apresentaram valores de $a$ de Cronbach superiores a 0,70 , que são considerados adequados ${ }^{10}$ : ilusão pelo trabalho $(\alpha=0,92)$, desgaste psíquico $(\alpha=0,88)$, indolência $(\alpha=0,89)$ e culpa $(\alpha=0,88)$.

Quanto às médias alcançadas em cada subescala, verifica-se na Tabela 4 que a subescala ilusão pelo trabalho apresentou média 
Tabela 1. Caracterização socioeconômica e demográfica da amostra de gestores de saúde do Estado de São Paulo, em 2013 ( $n=199)$

\begin{tabular}{|c|c|c|}
\hline \multirow{2}{*}{ Variáveis } & \multicolumn{2}{|c|}{ Amostra } \\
\hline & $N$ & $\%$ \\
\hline \multicolumn{3}{|l|}{ Gênero } \\
\hline Masculino & 77 & 38,7 \\
\hline Feminino & 122 & 61,3 \\
\hline \multicolumn{3}{|l|}{ Faixa etária } \\
\hline Abaixo de 35 anos & 46 & 23,1 \\
\hline De 35 anos a 50 anos & 104 & 52,3 \\
\hline Acima de 50 anos & 49 & 24,6 \\
\hline \multicolumn{3}{|l|}{ Estado Civil } \\
\hline Casado & 130 & 65,3 \\
\hline Solteiro, divorciado ou viúvo & 69 & 34,7 \\
\hline \multicolumn{3}{|l|}{ Número de filhos } \\
\hline Nenhum & 50 & 25,1 \\
\hline Um ou dois & 119 & 59,8 \\
\hline Três ou mais & 30 & 15,1 \\
\hline \multicolumn{3}{|l|}{ Grau de instrução } \\
\hline Superior incompleto & 26 & 13,6 \\
\hline Superior completo & 173 & 86,4 \\
\hline \multicolumn{3}{|l|}{ Renda familiar } \\
\hline Até 7 salários mínimos & 78 & 39,2 \\
\hline Acima de 7 salários mínimos & 121 & 60,8 \\
\hline \multicolumn{3}{|l|}{ Número de pessoas na casa } \\
\hline Até 2 & 60 & 30,1 \\
\hline 3 a 4 & 124 & 62,4 \\
\hline 5 ou mais & 15 & 7,5 \\
\hline \multicolumn{3}{|l|}{ Moradia } \\
\hline Residência própria & 162 & 81,4 \\
\hline Residência alugada/cedida & 37 & 18,6 \\
\hline
\end{tabular}

de $1,15(\mathrm{DP}=0,53)$. Essa subescala do CESQT, diferentemente das demais, possui itens formulados em sentido positivo, ou seja, altas pontuações indicam baixos níveis de $S B$. Dessa forma, o valor encontrado na amostra deste estudo é indicativo de $S B$. Por sua vez, a subescala desgaste psíquico apresentou média de 2,03 $(\mathrm{DP}=0,84)$, que também é valor indicativo de $S B$. Em contrapartida, as subescalas indolência e culpa apresentaram, respectivamente, média de $1,55(\mathrm{DP}=0,77)$ e $1,39(\mathrm{DP}=0,56)$, valores que não indicam presença de $\mathrm{SB}$.

De acordo com os critérios de classificação da $S B$ de Gil-Monte", foram aplicados P10, P33, P66 e P90 do manual do CESQT na amostra do estudo para cada dimensão (Tabela 5). Considerando as dimensões do CESQT, são considerados casos críticos em cada dimensão os 26 (13,1\%) gestores que apresentaram nível muito baixo de ilusão pelo trabalho, os $22(11,1 \%)$ que apresentaram nível crítico de desgaste psíquico, os 19 (9,5\%) que apresentaram nível crítico de indolência e os 19 (9,5\%) que apresentaram nível crítico de culpa. Os resultados finais apontaram que 9,5\% $(n=23)$ dos gestores avaliados apresentaram Perfil 1 da SB e que $3,5 \%(n=7)$ dos gestores encontravam-se no Perfil 2.

Tabela 2. Caracterização da amostra por profissões dos gestores de saúde do Estado de São Paulo, em 2013 (n=199)

\begin{tabular}{lcc}
\multicolumn{1}{c}{ Categorias profissionais } & $\boldsymbol{N}$ & $\boldsymbol{\%}$ \\
Profissões da área da saúde e afins & - & - \\
Enfermeiro & 59 & 29,7 \\
Cirurgião-dentista & 18 & 9,0 \\
Médico & 14 & 7,0 \\
Farmacêutico & 11 & 5,6 \\
Nutricionista & 6 & 3,0 \\
Assistente social & 6 & 3,0 \\
Auxiliar de enfermagem & 5 & 2,5 \\
Fisioterapeuta & 5 & 2,5 \\
Psicólogo & 3 & 1,5 \\
Fonoaudiólogo & 2 & 1,0 \\
Biomédico & 1 & 0,5 \\
Médico-veterinário & 1 & 0,5 \\
Técnico em patologia clínica & 1 & 0,5 \\
Total (A) & $\mathbf{1 3 2}$ & $\mathbf{6 6 , 3}$ \\
Outras profissões & - & - \\
Administrador & 21 & 10,6 \\
Gestor em saúde & 17 & 8,6 \\
Professor & 10 & 5,0 \\
Funcionário público & 9 & 4,5 \\
Advogado & 3 & 1,5 \\
Comerciante & 1 & 0,5 \\
Economista & 1 & 0,5 \\
Empresário & 1 & 0,5 \\
Engenheiro & 1 & 0,5 \\
Industrial & 1 & 0,5 \\
Técnico em estradas & 1 & 0,5 \\
Vigilante & $\mathbf{6 7}$ & 0,5 \\
Total (B) & & \\
\hline & 199 & $\mathbf{1 0 0}, 0$ \\
\hline
\end{tabular}

Tabela 3. Caracterização da amostra do trabalho como gestor em saúde do Estado de São Paulo, em 2013 (n=199)

\section{Variáveis}

Número de habitantes do município

Até $20 \mathrm{mil}$

De 20 mil a 100 mil

De 100 mil a 200 mil

Acima de $200 \mathrm{mil}$

Tempo de gestor

Menos de 1 ano

De 2 a 4 anos

De 5 a 10 anos

De 11 a 15 anos

De 16 a 20 anos

Mais de 20 anos

Horas trabalhadas

Até 8 horas/dia

De 8 a 12 horas/dia

Mais de 12 horas/dia

Exerce outra atividade?

\begin{tabular}{lcc} 
Sim & 69 & 34,7 \\
Não & 130 & 65,3 \\
\hline
\end{tabular}




\section{DISCUSSÃO}

O presente estudo oferece informações em relação à SB em gestores de saúde. Os dados obtidos são importante fonte de reflexão e debate para os que trabalham em saúde pública. Devido ao fato de este estudo ser inédito e não existirem outros estudos relacionados especificamente a esses profissionais, os dados obtidos nesta pesquisa se comparam a estudos semelhantes, mas com outros profissionais, especialmente da área da saúde $\mathrm{e}^{4,11-14} \mathrm{e}$ professores universitários ${ }^{15,16}$. Embora as circunstâncias laborais possam ser diferentes, há semelhanças no que se refere ao contato com pessoas e à dedicação exigida no exercício da função, com exposição intensa à tensão e ao estresse, o que propicia o aparecimento do esgotamento profissional ${ }^{17-19}$.

Os gestores municipais de saúde são submetidos a vários aspectos sociais ligados ao trabalho em geral, o que tem contribuído para elevar a carga de morbidade em trabalhadores, como a crescente intensidade das relações humanas no âmbito laboral, a pressão procedente das novas características econômicas próprias da globalização, a crescente competitividade, assim como a evidência de um incremento da violência psicológica nos ambientes de trabalho ${ }^{1,15}$. Considerando as consequências negativas do esgotamento profissional, buscar por subsídios para a compreensão e o controle das variáveis de risco ao esgotamento permite avançar na melhoria dos cuidados ofertados aos próprios gestores de saúde e aos usuários do SUS ${ }^{12}$.

O trabalho do gestor municipal em saúde exige grande responsabilidade, pois esse profissional lida diretamente com questões que envolvem a capacidade de resolução, tanto para as necessidades básicas quanto para a crescente complexidade dos

Tabela 4. Média, desvio-padrão (DP), variação e consistência interna ( $\alpha$ de Cronbach) das dimensões do CESQT

\begin{tabular}{lccc} 
& Média (DP) & Variação & $\begin{array}{c}\boldsymbol{\alpha} \text { de } \\
\text { Cronbach }\end{array}$ \\
$\begin{array}{l}\text { Ilusão pelo } \\
\text { trabalho }\end{array}$ & $1,15(0,53)$ & $0-4$ & 0,92 \\
$\begin{array}{l}\text { Desgaste psíquico } \\
\text { Indolência }\end{array}$ & $2,03(0,84)$ & $0-4$ & 0,88 \\
Culpa & $0,94(0,68)$ & $0-4$ & 0,89 \\
CESQT (15 itens) & $1,47(0,75)$ & $0-4$ & 0,88 \\
\hline
\end{tabular}

cuidados em saúde de uma população. Assim sendo, o acúmulo de funções dos gestores, a pressão para atender à demanda e melhorar a qualidade dos serviços oferecidos podem gerar uma sobrecarga de trabalho, levando a alterações de saúde e à insatisfação, o que afeta o processo e a qualidade do trabalho.

É importante enfatizar que a exaustão atinge cada indivíduo de forma única e que elicia diferentes respostas, fisiológicas e emocionais. Alguns ambientes de trabalho são propícios para desencadear estresse, em especial se o indivíduo não estiver munido de um repertório adequado de estratégias de enfrentamento. Geralmente, o que leva ao estresse laboral crônico ou a $S B$ é o desequilíbrio entre as demandas do trabalho e as capacidades de execução dessas demandas ${ }^{13,20}$.

O tempo é uma importante variável quando se estuda a $S B$, por se tratar de uma doença crônica. Mais da metade da amostra deste estudo exercia a gestão havia menos de um ano. Sabe-se que é no início do exercício profissional que os indivíduos se encontram em um período de sensibilização, quando a transição das expectativas idealistas para a realidade da prática cotidiana mostra que as primeiras nem sempre se realizam conforme prometido ou esperado ${ }^{21}$. Isso pode justificar o fato de os gestores da amostra terem apresentado baixo nível de ilusão pelo trabalho, indicativo da presença da síndrome ${ }^{8}$. A ilusão pelo trabalho é definida como a esperança de uma pessoa para se alcançar determinadas metas de trabalho ${ }^{8}$. Essa dimensão representa a exaustão emocional, marcada pela falta de energia, de empolgação, de entusiasmo e pelo sentimento de falência de recursos ${ }^{22}$.

Os gestores também apresentaram desgaste psíquico, outro indicativo da presença de $S B$. Foi possível observar a presença de exaustão física e emocional em virtude da atividade laboral, gerada pela relação diária com indivíduos que causam ou fazem aumentar o problema ${ }^{8}$. O gestor em saúde lida, constantemente, com o enfrentamento de problemas, ora tendo que atender às exigências de seus superiores, ora tendo que atender às demandas da população assistida e dos servidores, o que exige flexibilidade e capacidade de gerenciar conflitos.

No entanto, o gestor participa da elaboração e da administração de ações e de estratégias, e essa participação, com o exercício de sua criatividade e aptidão em solucionar problemas, pode

Tabela 5. Frequência absoluta e relativa de gestores municipais de saúde do Estado de São Paulo com níveis muito baixo, baixo, médio, alto e crítico da Síndrome de Burnout (SB) de acordo com os percentis (P) do Manual do CESQT

\begin{tabular}{lccccc} 
& Muito baixo & Baixo & Médio & Alto & Crítico \\
\cline { 2 - 6 } & $\boldsymbol{P} \leq \mathbf{1 0}$ & $\mathbf{P 1 1 - 3 3}$ & $\mathbf{P 3 4 - 6 6}$ & $\mathbf{P 6 7 - 8 9}$ & \multicolumn{1}{c}{ P } \\
Ilusão pelo trabalho & $26(13,1 \%)$ & $45(22,6 \%)$ & $90(45,2 \%)$ & $21(10,6 \%)$ & $17(8,5 \%)$ \\
Desgaste psíquico & $33(16,6 \%)$ & $30(15,1 \%)$ & $82(41,2 \%)$ & $32(16,1 \%)$ & $22(11,1 \%)$ \\
Indolência & $22(11,1 \%)$ & $49(24,6 \%)$ & $85(42,7 \%)$ & $24(12,1 \%)$ & $19(9,5 \%)$ \\
Culpa & $28(14,1 \%)$ & $42(21,1 \%)$ & $82(41,2 \%)$ & $28(14,1 \%)$ & $19(9,5 \%)$ \\
CESQT (15 itens) & $21(10,6 \%)$ & $45(22,6 \%)$ & $83(41,7 \%)$ & $27(13,6 \%)$ & $23(11,6 \%)$ \\
\hline
\end{tabular}


tornar o trabalho mais prazeroso ${ }^{23}$. Essa afirmação é corroborada pelos resultados do trabalho de Benevides-Pereira ${ }^{19}$, que relatou que quanto maior o comprometimento e a autonomia no desenvolvimento das atividades, maiores são os níveis de realização profissional e de satisfação. No presente estudo, a média apresentada pelos gestores da amostra para a subescala indolência não indicou a presença de $S B$. É possível, portanto, que os profissionais encarem o cargo de gestor como atraente, desafiador, e que interpretem o estresse laboral como positivo; consequentemente, como relatado por Carlotto ${ }^{24}$, a existência de um processo de instalação de Burnout em curso seja contida pelo sentimento de satisfação intrínseca com o trabalho desenvolvido.

Todavia, nem sempre o trabalho é fonte de realização profissional, podendo muitas vezes gerar problemas de insatisfação e exaustão ${ }^{11}$, pois a $S B$ também ocorre em trabalhadores altamente motivados ou satisfeitos, que reagem ao estresse laboral trabalhando ainda mais, até entrarem em colapso ${ }^{25}$, situação que aumenta a exigência de atenção para os primeiros sinais da presença da síndrome. Nesta amostra, quase um quarto dos gestores trabalhava por mais de $12 \mathrm{~h}$ por dia.

Outra variável que merece destaque é o nível de escolaridade, pois as pesquisas evidenciam que quanto mais alto ele for, maiores serão as chances de as pessoas apresentaram altos níveis de estresse ${ }^{19,26}$. A maioria dos participantes deste estudo informou, como grau de instrução, o nível superior completo.

Importante destacar também que quatro gestores apresentaram o Perfil 2 da síndrome, que é caracterizada por condutas e atitudes negativas no ambiente de trabalho, especialmente com aqueles com quem se deve ter um relacionamento profissional ${ }^{8}$. Ora, na atividade laboral própria dos serviços de saúde, nos quais se impõem exigências, tarefas e habilidades específicas para com a população, atenção especial deve ser dada aos profissionais que apresentam as manifestações da $S B^{13}$. Atitudes incompatíveis com o contexto do atendimento em saúde, tais como dificuldades de trabalhar em equipe, de respeitar os saberes dos outros profissionais e de atuar de forma empática com a clientela, podem prejudicar todo o processo de trabalho, resultando em baixa qualidade do serviço prestado.

Por todas as implicações e consequências para a saúde da $S B$, enfatiza-se a relevância de se executar uma busca sistematizada e permanente de trabalhadores adoecidos pela SB. É importante a notificação, como evento sentinela, dos casos de Burnout confirmados clinicamente, como parte dos transtornos mentais relacionados ao trabalho, conforme estabelece o Manual de Doenças Relacionadas ao Trabalho do Ministério da Saúde ${ }^{27}$, seguido de investigação mais criteriosa das condições de trabalho ${ }^{14}$.

Vale ressaltar que, obedecendo às exigências com relação à ética em pesquisa com seres humanos, a participação no presente estudo foi totalmente voluntária. Dessa forma, é possível que isso tenha levado a um viés de seleção dos indivíduos da amostra, pois, devido às características da SB e à forma como ela evolui clinicamente, pode ser que os gestores mais seriamente afetados não tenham participado. Costa et al. ${ }^{16}$, em pesquisa com professores universitários, também levantaram essa hipótese.

Os valores de $\alpha$ de Cronbach encontrados no presente estudo são considerados adequados de acordo com Carretero-Dios e Pérez ${ }^{10}$. Portanto, é possível afirmar que todos os itens estão relacionados significativamente com seus construtos originais para avaliar a mesma faceta da $S B$. Esses resultados também foram obtidos por Gil-Monte et al. ${ }^{8}$ no estudo de avaliação da validade fatorial e de construto da versão brasileira do CESQT, por Gil-Monte et al. ${ }^{9}$ em uma amostra de docentes da região Sul do Brasil e por Costa et al. ${ }^{16}$ em uma amostra de professores universitários brasileiros.

\section{CONSIDERAÇÕES FINAIS}

A amostra de gestores municipais apresentou baixa prevalência de $S B$. Todavia, por tratar-se de um estudo inédito, os resultados levam para novas questões, em vez de levarem a conclusões definitivas. Sugere-se, portanto, como continuidade do presente estudo, replicar a coleta de dados, entre os mesmos gestores, passados alguns anos na gestão, bem como buscar um aprofundamento das questões por meio de abordagens qualitativas, que podem ser úteis para melhor elucidar significados e percepções dos sujeitos.

Novos estudos relacionados à $S B$ utilizando o instrumento CESQT entre os gestores municipais em saúde em outras regiões do país também seriam pertinentes. Consideramos que, em razão da relevância do cargo de gestor municipal para manutenção dos princípios do SUS e as consequências que a $S B$ pode acarretar tanto para o indivíduo como para todo o sistema, aprofundar o conhecimento na área em busca de respostas que levem à prevenção e controle da síndrome é de vital importância.

\section{REFERÊNCIAS}

1. Barbosa RMDSA, Guimarães TA. Síndrome de Burnout: relações com comprometimento afetivo entre gestores de organização estatal. RAM: Revista de Administração Mackenzie. 2008;6(1):157-79.

2. Brasil. Ministério da Saúde. Secretaria de Gestão Estratégica e Participativa. Política Nacional de Gestão Estratégica e Participativa no SUS [Internet]. 2. ed. Brasília: MS; 2009 [citado em 2013 Jun 22]. Disponível em: http://bvsms. saude.gov.br/bvs/publicacoes/politica_estrategica_participasus_2ed.pdf

3. Tamayo MR, Tróccoli BT. Burnout no trabalho. In: Mendes AM, Borges LO, Ferreira MC, organizadores. Trabalho em transição, saúde em risco. Brasília: Universidade de Brasília; 2002.

4. Moreira DS, Magnago RF, Sakae TM, Magajewski FRL. Prevalência da síndrome de burnout em trabalhadores de enfermagem de um hospital de grande porte da Região Sul do Brasil. Cad Saude Publica. 2009;25(7):155968. http://dx.doi.org/10.1590/S0102-311X2009000700014. PMid:19578577. 
5. Maslach C, Jackson SE. The measurement of experienced burnout. J Organ Behav. 1981;2(2):99-113. http://dx.doi.org/10.1002/job.4030020205.

6. Gil-Monte PR. El síndrome de quemarse por el trabajo ("burnout"): uma efermedad laboral em La sociedad del bienestar. Madrid: Pirámide; 2005.

7. Meneghim MC, Kozlowski FC, Pereira AC, Ambrosano GMB, Meneghim ZMAP. Classificação socioeconômica e sua discussão em relação à prevalência de cárie e fluorose dentária. Cien Saude Colet. 2007;12(2):523-9. http:// dx.doi.org/10.1590/S1413-81232007000200028. PMid:17680106.

8. Gil-Monte PR, Carlotto MS, Câmara SG. Validação da versão brasileira do "Cuestionario para la Evaluación del Síndrome de Quemarse por el Trabajo" em professores. Rev Saude Publica. 2010;44(1):140-7. http:// dx.doi.org/10.1590/S0034-89102010000100015. PMid:20140338.

9. Gil-Monte PR. CESQT - Cuestionario para la Evalución del Síndrome de Quemarse por el Trabajo: manual. Madrid: TEA; 2011.

10. Carretero-Dios H, Pérez C. Standards for the development na review of instrumental studies: considerations about test selection in psychological research. Int J Clin Health Psychol. 2007;7(3):863-82.

11. Lima RAS, Souza AI, Galindo RH, Feliciano KVO. Vulnerabilidade ao burnout entre médicos de hospital público do Recife. Cien Saude Colet. 2013;18(4):1051-8. http://dx.doi.org/10.1590/S1413-81232013000400018. PMid:23670382.

12. Martins LF, Laport TJ, Menezes VP, Medeiros PB, Ronzani TM. Esgotamento entre profissionais da atenção primária à saúde. Cien Saude Colet. 2014;19(12):4939-4750. http://dx.doi.org/10.1590/1413812320141912.03202013. PMid:25388182.

13. Silva SCPS, Nunes MAP, Santana VR, Reis FP, Machado No J, Lima SO. A síndrome de burnout em profissionais da Rede de Atenção Primária à Saúde de Aracaju, Brasil. Cien Saude Colet. 2015, 20(10):3011-3020, 2015.

14. Ferreira NN, Lucca SR. Síndrome de burnout em técnicos de enfermagem de um hospital público do Estado de São Paulo. Rev Bras Epidemiol. 2015;18(1):68-79. http://dx.doi.org/10.1590/1980-5497201500010006. PMid:25651012.

15. Carlotto MS, Palazzo LS. Sìndrome de Burnout e fatores associados: um estudo epidemiológico com professores. Cad Saude Publica. 2006; 22(5):1017-26.
16. Costa LST, Gil-Monte PR, Possobon RF, Ambrosano GMB. Prevalência da Síndrome de Burnout em uma amostra de professores universitários brasileiros. Psicol Reflex Crit. 2013;26(4):636-42. http://dx.doi.org/10.1590/ S0102-79722013000400003.

17. Maslach C, Jackson SE, Leiter M. Maslach burnout inventory: manual. 3. ed. Palo Alto: Consulting Psychologists Press; 1996.

18. Codo W, coordenador. Educação: carinho e trabalho: burnout, a síndrome da desistência do educador, que pode levar à falência da educação. 3. ed. Rio de Janeiro: Vozes; 2002.

19. Benevides-Pereira AMT. Burnout: quando o trabalho ameaça o bem-estar do trabalhador. São Paulo: Casa do Psicólogo; 2002.

20. Alpi VS, Quiceno JM. Burnout: "Síndrome de Quemarse en el Trabajo (SQT). Acta Colomb Psicol. 2007;10(2):117-25.

21. Martínez JCA. Aspectos epidemiológicos del síndrome de Burnout en personal sanitario. Rev Esp Salud Publica. 1997;71(3):293-303. http:// dx.doi.org/10.1590/S1135-57271997000300008. PMid:9445757.

22. Maslach C, Schaufeli WB, Leiter MP. Job Burnout. Annu Rev Psychol 2001;52(1):397-422. http://dx.doi.org/10.1146/annurev.psych.52.1.397. PMid:11148311.

23. Dejours C. A loucura do trabalho: estudo da psicopatologia do trabalho. São Paulo: Oboré; 1994.

24. Carlotto MS. A Síndrome de burnout e o trabalho docente. Psicol Estud. 2002;7(1):21-9. http://dx.doi.org/10.1590/S1413-73722002000100005.

25. Tamayo MR. Relação entre síndrome de burnout e os valores organizacionais no pessoal de enfermagem de dois hospitais públicos [dissertação]. Brasília: Universidade de Brasília, Instituto de Psicologia; 1997.

26. Carlotto MS. Síndrome de burnout diferenças segundo níveis de ensino. PSICO. 2010;41(4):495-502.

27. Brasil. Ministério da Saúde. Organização Pan-Americana da Saúde no Brasil. Doenças relacionadas ao trabalho: manual de procedimentos para os serviços de saúde [Internet]. Brasília: MS; 2001 [citado em 2013 Jun 22]. Disponível em: http://bvsms.saude.gov.br/bvs/publicacoes/doencas_ relacionadas_trabalho1.pdf

Recebido em: Jan. 19, 2016 Aprovado em: Abr. 25, 2016 